\title{
On finite simple groups and Kneser graphs
}

\author{
Andrea Lucchini • Attila Maróti
}

Received: 15 October 2008 / Accepted: 5 March 2009 / Published online: 20 March 2009

(C) Springer Science+Business Media, LLC 2009

\begin{abstract}
For a finite group $G$ let $\Gamma(G)$ be the (simple) graph defined on the elements of $G$ with an edge between two (distinct) vertices if and only if they generate $G$. The chromatic number of $\Gamma(G)$ is considered for various non-solvable groups $G$.
\end{abstract}

Keywords Kneser graph · Chromatic number · Finite simple group · Special linear group $\cdot$ Symmetric group

\section{Introduction}

For a finite non-cyclic group $G$ let $\sigma(G)$ denote the least number of proper subgroups of $G$ whose union is $G$. The function $\sigma(G)$ has been much investigated. For example, Tomkinson [25] showed that $\sigma(G)=|V|+1$ for a solvable group $G$ where $V$ denotes the smallest chief factor of $G$ which has more than one complement.

Let $G$ be a finite group that can be generated by two elements. A subset $S$ of $G$ is said to pairwise generate $G$ if every distinct pair of elements of $S$ generates $G$.

Research of the second author was supported by OTKA NK72523, OTKA T049841, NSF Grant DMS 0140578, and by a fellowship of the Mathematical Sciences Research Institute.

\footnotetext{
A. Lucchini

Dipartimento di Matematica Pura ed Applicata, Via Trieste 63, 35121 Padova, Italy e-mail: lucchini@math.unipd.it
}

\section{A. Maróti $(\bowtie)$}

Alfréd Rényi Institute of Mathematics, Hungarian Academy of Sciences, Reáltanoda utca 13-15,

1053, Budapest, Hungary

e-mail: maroti@renyi.hu

\section{A. Maróti}

Department of Mathematics, University of Southern California, Los Angeles, CA 90089-1113, USA

e-mail: maroti@usc.edu 
The maximal size of a pairwise generating set in $G$ is denoted by $\omega(G)$. Clearly, $\omega(G) \leq \sigma(G)$ if both invariants are defined.

Blackburn [1] showed that $\omega(\operatorname{Sym}(n))=\sigma(\operatorname{Sym}(n))=2^{n-1}$ for sufficiently large odd $n$ and $\omega(\operatorname{Alt}(n))=\sigma(\operatorname{Alt}(n))=2^{n-2}$ for sufficiently large even $n$ not divisible by 4 . In the same paper Blackburn asked whether $\omega(G) / \sigma(G)$ tends to 1 as the size of the non-abelian finite simple group $G$ tends to infinity. Stringer [23] proved that the answer is affirmative for alternating groups and Britnell, Evseev, Guralnick, Holmes, Maróti [3] showed that the answer is affirmative for projective special linear groups.

Stringer's [23] and Maróti's [21] results imply that there exists a constant $c \geq 1$ such that $(1-c / n) \sigma(\operatorname{Alt}(n)) \leq \omega(\operatorname{Alt}(n))$ for $n$ not divisible by 4 and not a prime of the form $\left(q^{k}-1\right) /(q-1)$ where $q$ is a prime power and $k$ is a positive integer. For a finite group $G$ let $m(G)$ be the minimal index of a proper subgroup in $G$. Clearly, $m(\operatorname{Alt}(n))=n$ for $n>1$. Our first result is

Theorem 1.1 There exists a universal constant $c \geq 1$ such that if $G$ is a projective special linear group, a Suzuki group, or a Ree group, then $(1-c / m(G)) \sigma(G) \leq$ $\omega(G)$.

The question arises: does there exist a universal constant $c \geq 1$ such that if $G$ is a non-abelian finite simple group, then $(1-c / m(G)) \sigma(G) \leq \omega(G)$ ? The answer is not known even for alternating groups. The case of special linear groups and Suzuki groups show that $c$ cannot be taken to be less than 1 .

For a finite group $G$ let $\Gamma(G)$ be the (simple) graph defined on the elements of $G$ with an edge between two (distinct) vertices if and only if they generate $G$. Let the chromatic number (least number of colors needed to color the vertices of the graph in such a way that the endpoints of every edge receive different colors) of the graph $\Gamma(G)$ be $\chi(G)$. Clearly, $\omega(G) \leq \chi(G) \leq \sigma(G)$ provided that all three invariants are defined.

In the proof of Theorem 1.1 the chromatic numbers $\chi(G)$ are calculated for various linear groups and Suzuki groups $G$. For linear groups $G$ of large dimensions there is a formula for $\sigma(G)$ (see [3]). In this paper the following is shown.

Theorem 1.2 Let $n$ be a positive integer at least 12 and let $q$ be a prime power. Let $G$ be any of the groups $(\mathrm{P}) \mathrm{GL}(n, q),(\mathrm{P}) \operatorname{SL}(n, q)$. Then

$$
\omega(G)-1 \leq\left(\frac{10}{q^{n / 2}-1}\right) \omega(G)+\left(\frac{q^{n / 2}-11}{q^{n / 2}-1}\right) \sigma(G)-1<\chi(G) \leq \sigma(G) .
$$

Note that in the formula (above) in the statement of Theorem 1.2 only the second inequality is 'new'.

In the proofs of Theorems 1.1 and 1.2 certain variants of Kneser graphs appear.

Let $r$ and $n$ be positive integers with $r$ not greater than $n$. The Kneser graph $K(n, r)$ is the graph whose vertices are the $r$-element subsets of a set of size $n$ and there is an edge between two subsets if and only if they are disjoint. Kneser conjectured that the chromatic number of $K(n, r)$ is $n-2 r+2$. This was proved by Lovász in [17]. There are many papers on Kneser graphs. Here the following result is shown. 
For a positive real number $x$, the base $2 \operatorname{logarithm}$ is denoted by $\log x$ and the natural (base $e$ ) logarithm is denoted by $\ln x$.

Theorem 1.3 Let $r$ and $n$ be positive integers so that $r<n / 2$. There exists a positive constant $c$ so that whenever $2 r \log r+2 r+c<n$ then

(1) the Kneser graph $K(n, r)$ is an induced subgraph of $\Gamma(\operatorname{Sym}(n))$ for all even $n-r$

(2) the Kneser graph $K(n, r)$ is an induced subgraph of $\Gamma(\operatorname{Alt}(n))$ for all odd $n-r$.

Let $F$ be a finite field of order $q$. The $q$-Kneser graph $q K(n, r)$ is the graph whose vertices are the $r$-dimensional subspaces of an $n$-dimensional vector space over $F$ and two vertices are connected by an edge if and only if their intersection is trivial. The chromatic number of the graph $q K(n, r)$ is investigated in [5]. In view of the present investigations the question arises: for a fixed $r$ is $q K(n, r)$ an induced subgraph of $\Gamma(\mathrm{GL}(n, q))$ for sufficiently large $n$ ?

For a finite group $G$ let $P(G)$ be the probability that two random elements of $G$ generate $G$. In [16] Liebeck and Shalev proved that there exist constants $c_{1}, c_{2}>0$ such that $1-\left(c_{1} / m(G)\right) \leq P(G) \leq 1-\left(c_{2} / m(G)\right)$ for all non-abelian finite simple groups $G$. Two consequences of this theorem are the following.

Theorem 1.4 There exists a universal positive constant d so that

$$
P(G) \leq 1-\frac{d}{|G|^{1 / 3}}
$$

for a non-solvable finite group $G$.

Theorem 1.5 There exist positive constants $d_{1}$ and $d_{2}$ so that for any non-abelian finite simple group $G$ the graph $\Gamma(G)$ contains (as subgraphs) every r-colorable graph on at most $d_{2} m(G)(\log |G| / \log m(G))$ vertices for $r \leq d_{1} m(G)$.

Theorem 1.5 is a slight extension of the Liebeck-Shalev result [16] stating that there exists a universal constant $c>0$ so that $c \cdot m(G) \leq \omega(G)$ for a non-abelian finite simple group $G$.

Finally, the paper culminates in a proof of the following theorem.

Theorem 1.6 Let $\alpha$ denote $\omega, \chi$, or $\sigma$. For a positive number $x$ define $\alpha(x)$ to be the number of positive integers $n$ at most $x$ with the property that there exists a non-abelian finite simple group $G$ so that $\alpha(G)=n$. Then $\alpha(x)$ is equal to $(2 \sqrt{2}+o(1))(\sqrt{x} / \ln x)$.

\section{Linear groups of dimension 2}

Throughout this section let $q=p^{f}$ be a prime power where $p$ is a prime and $f$ is a positive integer. Also, in this section $G$ will always denote any of the groups 
$\operatorname{GL}(2, q), \operatorname{SL}(2, q), \operatorname{PGL}(2, q), \operatorname{PSL}(2, q)$. We seek to determine $\omega(G), \chi(G)$, and $\sigma(G)$.

We begin by stating Dickson's [8] result about the maximal subgroups of $\operatorname{PSL}(2, q)$. The result is divided according to the parity of $p$.

Theorem 2.1 (Dickson, [8]) Let $q=2^{f} \geq 4$. Then the maximal subgroups of $\operatorname{PSL}(2, q)$ are

(1) $C_{2}{ }^{f} \rtimes C_{q-1}$, that is, the stabilizer of a point of the projective line;

(2) $D_{2(q-1)}$;

(3) $D_{2(q+1)}$;

(4) PGL(2, $\left.q_{0}\right)$ where $q=q_{0}^{r}$ for some prime $r$ and $q_{0} \neq 2$.

Theorem 2.2 (Dickson, [8]) Let $q=p^{f} \geq 5$ with $p$ an odd prime. Then the maximal subgroups of $\operatorname{PSL}(2, q)$ are

(1) $C_{p}{ }^{f} \rtimes C_{(q-1) / 2}$, that is, the stabilizer of a point of the projective line;

(2) $D_{q-1}$ for $q \geq 13$;

(3) $D_{q+1}$ for $q \neq 7,9$;

(4) $\operatorname{PGL}\left(2, q_{0}\right)$ for $q=q_{0}^{2}$ (two conjugacy classes);

(5) $\operatorname{PSL}\left(2, q_{0}\right)$ for $q=q_{0}{ }^{r}$ where $r$ is an odd prime;

(6) Alt(5) for $q \equiv \pm 1(\bmod 10)$ where either $q=p$ or $q=p^{2}$ and $p \equiv \pm 3$ ( $\bmod 10)$ (two conjugacy classes);

(7) Alt(4) for $q=p \equiv \pm 3(\bmod 8)$ and $q \not \equiv \pm 1(\bmod 10)$;

(8) $\operatorname{Sym}(4)$ for $q=p \equiv \pm 1(\bmod 8)$ (two conjugacy classes).

We next state the corresponding result about the maximal subgroups of $\operatorname{PGL}(2, q)$ when $q$ is odd. (For $q$ even we have $\operatorname{SL}(2, q) \cong \operatorname{PSL}(2, q)$ and $\operatorname{GL}(2, q) \cong \operatorname{SL}(2, q) \times$ $C_{q-1}$, hence $\left.\operatorname{PGL}(2, q) \cong \operatorname{PSL}(2, q)\right)$.

Theorem 2.3 Let $G=\operatorname{PGL}(2, q)$ with $q=p^{f}>3$ for some odd prime $p$. Then the maximal subgroups of $G$ not containing $\operatorname{PSL}(2, q)$ are

(1) $C_{p}^{f} \rtimes C_{q-1}$;

(2) $D_{2(q-1)}$ for $q \neq 5$

(3) $D_{2(q+1)}$;

(4) $\operatorname{Sym}(4)$ for $q=p \equiv \pm 3(\bmod 8)$;

(5) $\operatorname{PGL}\left(2, q_{0}\right)$ for $q=q_{0}{ }^{r}$ with $r$ an odd prime.

Only in this paragraph let $G$ be $\operatorname{GL}(2, q)$ or $\operatorname{SL}(2, q)$, and denote the center of $G$ by $Z$. We claim that for any maximal subgroup $M$ of $G$ we have $Z \leq M$ or $\operatorname{SL}(2, q) \leq M$ whenever $q \geq 4$. For if $M$ is a maximal subgroup of $G$ not containing $Z$, then $M Z=G, M \triangleleft G$ and $G / M$ is abelian. It follows that $M$ contains the commutator subgroup of $G$ which is $\operatorname{SL}(2, q)$ for $q \geq 4$.

Let $V$ be a 2-dimensional vector space over the field of $q$ elements. The group $G$ acts naturally on the projective line $\mathcal{P}(1, q)$ thought of as the set of 1-dimensional subspaces of $V$. Let us assign an arbitrary labelling $\{1, \ldots, q+1\}$ to the points of $\mathcal{P}(1, q)$. We denote the stabilizer of the point $i$ in $G$ by $P_{i}$. Each group $P_{i}$ is maximal 
in $G$. Let us denote the center of $G$ by $Z$. A Singer cycle of $\operatorname{GL}(2, q)$ is a cyclic group of order $q^{2}-1$. Every Singer cycle acts irreducibly on $V$. The Singer cycles form a single conjugacy class in $\operatorname{GL}(2, q)$. Singer cycles are self-centralizing, and $Z$ is in every Singer cycle. If $S$ is a Singer cycle of GL $(2, q)$, then we will term $S \cap \operatorname{SL}(2, q)$, $(S \cap \operatorname{SL}(2, q)) /(Z \cap \operatorname{SL}(2, q))$, and $S / Z$ Singer cycles of $\operatorname{SL}(2, q), \operatorname{PSL}(2, q)$, and $\operatorname{PGL}(2, q)$, respectively. We know that $|S \cap \operatorname{SL}(2, q)|=q+1$. Also, whenever $D$ is a Singer normalizer in $\operatorname{GL}(2, q)$, then $D \cap \operatorname{SL}(2, q),(D \cap \operatorname{SL}(2, q)) /(Z \cap \operatorname{SL}(2, q))$, and $D / Z$ are Singer normalizers in $\operatorname{SL}(2, q), \operatorname{PSL}(2, q)$, and $\operatorname{PGL}(2, q)$, respectively. The order of the normalizer of a Singer cycle $S$ is $2|S|$, and the number of Singer cycles is $q(q-1) / 2$ whichever of the four groups $G$ might be. The set of all normalizers of Singer cycles together with all the $P_{i}$ groups forms a covering for $G$. (A covering is a collection of proper subgroups whose union is the group.) When $q$ is odd, denote this set of proper subgroups of $G$ by $\Sigma$. Furthermore, if $q$ is even, then the set of all normalizers of all Singer cycles together with all but one (say $P_{q+1}$ ) of the $P_{i}$ 's forms a covering for $G$. When $q$ is even, denote this set of proper subgroups of $G$ by $\Sigma$. Notice that the sizes of these sets are $(q(q+1) / 2)+1$ and $q(q+1) / 2$ respectively. These give us upper bounds for $\sigma(G)$. In fact, when $q$ is large enough, these upper bounds are exact.

Theorem 2.4 (Bryce, Fedri, Serena, [4]) Let $q \geq 4$ but different from 5, 7, 9 and let $G$ be any of the groups (P) $\mathrm{GL}(2, q),(\mathrm{P}) \mathrm{SL}(2, q)$. If $q$ is even, then $\sigma(G)=q(q+$ 1) $/ 2$. If $q$ is odd, then $\sigma(G)=(q(q+1) / 2)+1$.

Usually two generators of two distinct Singer cycles of $G$ generate $G$. From now on (in this section) this observation will be used extensively.

Lemma 2.1 (Lemma 3.3 of [4]) Let $q \geq 4$ and let $G$ be any of the groups $(\mathrm{P}) \mathrm{GL}(2, q),(\mathrm{P}) \mathrm{SL}(2, q)$. Then the normalizer of a Singer cycle $S$ is the unique maximal subgroup of $G$ containing $S$ except when $G=(\mathrm{P}) \operatorname{SL}(2, q)$ and $q=5,7$, or 9.

In some cases we can immediately determine $\omega(G)$ and $\chi(G)$.

Theorem 2.5 Let $q>9$ be an odd prime power. Let $G$ be any of the groups $\operatorname{PSL}(2, q), \operatorname{SL}(2, q)$. Then $\omega(G)=\chi(G)=\sigma(G)=(q(q+1) / 2)+1$.

Proof By Theorem 2.4 it is sufficient to show $(q(q+1) / 2)+1 \leq \omega(G)$.

Consider the set $X=\left\{s_{1}, \ldots, s_{t}, p_{1}, \ldots, p_{q+1}\right\}$ where the $s_{i}$ 's are generators for the distinct Singer cycles of $G$ and the $p_{i}$ 's are such $p$-elements from the $P_{i}$ 's which cannot be represented over any subfield of $G F(q)$. Clearly, $t=q(q-1) / 2$. It is sufficient to show that $X$ is a clique in $\Gamma(G)$ provided that $q>9$ is odd.

By Lemma 2.1, any $s_{i}$ is connected to any other element of $X$ in $\Gamma(G)$. Let $i \neq j$ be an arbitrary pair of distinct indices. Consider the subgroup $H=\left\langle p_{i}, p_{j}\right\rangle$ of $G$. Inspection shows that, since $q>9$, the subgroup $H$ is not contained in any of the maximal subgroups of $G$ of types (2), (3), (6), (7), (8) of Theorem 2.2. By the choice of $p_{i}$, the subgroup $H$ cannot be contained in a maximal subgroup of type (4) or (5) 
of Theorem 2.2. Finally, since $H$ is irreducible, it cannot be contained in any of the $P_{k}$ 's.

The proof of the above theorem suggests a way to establish lower bounds for $\omega(G)$ and $\chi(G)$ in the rest of the cases. Before we proceed we need two lemmas.

Lemma 2.2 Let $n$ be a positive integer at least 2. Let $\mathcal{A}$ be a family of distinct subsets of $\{1, \ldots, n\}$ each of size 2 such that each pair of subsets in $\mathcal{A}$ intersects non-trivially. If $|\mathcal{A}| \neq 1,3$, then $\left|\bigcap_{A \in \mathcal{A}} A\right|=1$.

Proof Put $m=|\mathcal{A}|$. The claim can be checked easily for $m \leq 3$, so suppose that $m \geq 4$. Without loss of generality suppose that $A_{1}=\{1,2\} \in \mathcal{A}$, that $A_{1}, \ldots, A_{k}$ are the distinct subsets in $\mathcal{A}$ containing 1 for some $k$ such that $m / 2 \leq k \leq m$, and $A_{k+1}, \ldots, A_{m}$ are the distinct subsets in $\mathcal{A}$ containing 2 but not 1 . We have to show that $k=m$. Suppose that $k<m$. Then $A_{m}=\{2, j\}$ for some $j>2$. But then $k=2$, $m=4$ and $A_{m-1}=A_{m}$. A contradiction.

Lemma 2.3 Let $n$ be a positive integer at least 3 . Let $K(n, 2)$ be the Kneser graph whose vertices are the 2-element subsets of $\{1, \ldots, n\}$ and two vertices are connected by an edge if and only if their intersection is trivial. Then $\omega(K(n, 2))=[n / 2]$ and $\chi(K(n, 2))=n-2$.

Proof Clearly, there is a clique of size $[n / 2]$ in $K(n, 2)$. On the other hand, if $Y$ is a clique in $K(n, 2)$, then the union of all vertices of $Y$ is a subset of $\{1, \ldots, n\}$ of size $2|Y|$. This proves $\omega(K(n, 2))=[n / 2]$.

For each $4 \leq i \leq n$ label all vertices of $K(n, 2)$ that contain $i$ as their largest element by $i$. Label all other vertices of $K(n, 2)$ by 3 . This way we get a good coloring of the vertices of $K(n, 2)$ by $n-2$ colors. This proves $\chi(K(n, 2)) \leq n-2$.

We claim that the set of vertices of $K(n, 2)$ is the union of no fewer than $n-2$ empty induced subgraphs of $K(n, 2)$ each of which is maximal with respect to inclusion. What are the empty induced subgraphs of $K(n, 2)$ that are maximal with respect to inclusion? Let $\mathcal{A}$ be a set of vertices of $K(n, 2)$ which together define an empty induced subgraph in $K(n, 2)$. Suppose also that $\mathcal{A}$ is maximal satisfying this property with respect to inclusion. By Lemma 2.2, if $|\mathcal{A}| \neq 3$, then $\mathcal{A}$ is equal to the set of all vertices of $K(n, 2)$ which contain a fixed positive integer, say $i$. Let us denote this particular set of vertices by $\mathcal{A}_{i}$. On the other hand, if $|\mathcal{A}|=3$, then $n \geq 3$ and there exist positive integers $i, j, k$ such that $1 \leq i<j<k \leq n$ and $\mathcal{A}=\{\{i, j\},\{i, k\},\{j, k\}\}$. Let this particular set be denoted by $\mathcal{A}_{i, j, k}$. The vertex-set of $K(n, 2)$ is the union of some of the $\mathcal{A}_{i}$ 's and some of the $\mathcal{A}_{i, j, k}$ 's. Suppose that the number of $\mathcal{A}_{i}$ 's in the covering is $\alpha$ and the number of $\mathcal{A}_{i, j, k}$ 's in the covering is $\beta$. Consider the induced subgraph of $K(n, 2)$ defined by those vertices of $K(n, 2)$ which are not contained in any of the $\mathcal{A}_{i}$ 's involved in the covering. This subgraph is isomorphic to $K(n-\alpha, 2)$. Since the vertex-set of $K(n-\alpha, 2)$ is contained in the union of $\beta$ sets each of order 3 , we have $\left(\begin{array}{c}n-\alpha \\ 2\end{array}\right) \leq 3 \beta$. From this we see that $n-2 \leq \alpha+((n-\alpha)(n-\alpha-1) / 6) \leq \alpha+\beta$ which proves $n-2 \leq \chi(K(n, 2))$.

From now on let us exclude the case when $G=(\mathrm{P}) \operatorname{SL}(2, q)$ and $q$ is odd. 
If $G=\mathrm{GL}(2, q)$, then for any distinct pair of indices $i$ and $j(1 \leq i<j \leq q+1)$ there exists a cyclic group $K_{i, j}$ of order $q-1$ such that $K_{i, j} Z=P_{i} \cap P_{j}$ and $K_{i, j}$ fixes every element of the $i$-th subspace. If $G=\operatorname{PGL}(2, q)$, then define $K_{i, j}$ to be the image of the subgroup $K_{i, j}$ of $\operatorname{GL}(2, q)$ in $\operatorname{PGL}(2, q)$. If $G=\operatorname{SL}(2, q)$, then for any distinct pair of indices $i$ and $j(1 \leq i<j \leq q+1)$ there exists a cyclic group $K_{i, j}$ of order $q-1$ such that $K_{i, j}=P_{i} \cap P_{j}$. Let $k_{i, j}$ be a fixed generator of $K_{i, j}$.

Consider the set $X$ consisting of all the above chosen $k_{i, j}$ 's together with the elements $s_{1}, \ldots, s_{t}$ where $t$ is the number of distinct Singer cycles in $G$ and $s_{i}$ denotes an arbitrarily chosen generator of the $i$-th Singer cycle of $G$. Let us view $X$ also as the induced subgraph of $\Gamma(G)$ determined by the vertex-set $X$. Clearly, $\omega(X) \leq \omega(G)$ and $\chi(X) \leq \chi(G)$.

For $q \geq 4$ any $s_{i}$ is connected (in $\Gamma(G)$ ) with any other element of $X$. (This follows from Lemma 2.1.) Let $k_{i, j}$ and $k_{\ell, r}$ be two distinct arbitrary elements of $X$ both leaving exactly two 1 -dimensional subspaces invariant. If $q \geq 4$, then $k_{i, j}$ and $k_{\ell, r}$ are connected by an edge if and only if $\{i, j\} \cap\{\ell, r\}=\emptyset$. (This follows from Theorems 2.1 and 2.3 and from the comments made after Theorem 2.3.)

Theorem 2.6 Let $q \geq 4$ and let $G$ be any of the groups (P) $\mathrm{GL}(2, q),(\mathrm{P}) \operatorname{SL}(2, q)$. Let us exclude the case when $G=(\mathrm{P}) \operatorname{SL}(2, q)$ and $q$ is odd. Let $X$ be as above. Then $\omega(X)=\left[\left(q^{2}+1\right) / 2\right]$. If $q$ is even, then

$$
\chi(X)=\chi(G)=\sigma(G)-1=(q(q+1) / 2)-1 .
$$

If $q$ is odd, then

$$
(q(q+1) / 2)-1=\chi(X) \leq \chi(G) \leq \sigma(G)=(q(q+1) / 2)+1 .
$$

Proof Let $X_{k}$ be the subgraph of $X$ induced by the vertices that do not correspond to generators of Singer cycles, and let $X_{s}$ be the subgraph of $X$ induced by the vertices that correspond to generators of Singer cycles. The graph $X_{s}$ is complete. Since every vertex of $X_{s}$ is connected to every vertex of $X_{k}$, it is clear that $\omega(X)=\omega\left(X_{s}\right)+\omega\left(X_{k}\right)=(q(q-1) / 2)+\omega\left(X_{k}\right)$ and $\chi(X)=\chi\left(X_{s}\right)+\chi\left(X_{k}\right)=$ $(q(q-1) / 2)+\chi\left(X_{k}\right)$. Hence it is sufficient to show that $\omega\left(X_{k}\right)=[(q+1) / 2]$, that $\chi\left(X_{k}\right)=q-1$ and that $\chi(G) \leq \chi(X)$ when $q$ is even, provided that $q \geq 4$.

Let $n$ be a positive integer at least 3 and let $K(n, 2)$ be the graph whose vertices are the distinct 2-element subsets of $\{1, \ldots, n\}$ with two vertices $A$ and $B$ connected by an edge if and only if $A \cap B=\emptyset$. Clearly, $X_{k} \cong K(q+1,2)$ provided that $q \geq 4$. By Lemma 2.3, we have $\omega(K(q+1,2))=[(q+1) / 2]$ and $\chi(K(q+1,2))=q-1$.

To complete the proof of the theorem we need to show that $\chi(G) \leq(q(q+1) / 2)-$ 1 when $q \geq 4$ is even. Set $\widetilde{\Sigma}=\left(\Sigma \backslash\left\{P_{q-1}, P_{q}\right\}\right) \cup\{P\}$ where $P$ is the set of all elements of $G$ leaving exactly two 1-dimensional subspaces invariant both of which are labelled by the positive integers $q-1, q$, or $q+1$. Clearly, $|\widetilde{\Sigma}|=(q(q+1) / 2)-1$, every element of $G$ is contained in some member of $\widetilde{\Sigma}$, and every member of $\widetilde{\Sigma}$ induces an empty subgraph in $\Gamma(G)$. This completes the proof of the theorem.

We now turn to the determination of $\omega(G)$. Recall the definition of $\Sigma$ (which depends on the parity of $q)$. If $q$ is odd, then $|\Sigma|=(q(q+1) / 2)+1$. If $q$ is even, then $|\Sigma|=q(q+1) / 2$. 
Theorem 2.7 Let $q \geq 4$ and let $G$ be any of the groups $(\mathrm{P}) \mathrm{GL}(2, q),(\mathrm{P}) \mathrm{SL}(2, q)$. Let us exclude the case when $G=(\mathrm{P}) \operatorname{SL}(2, q)$ and $q$ is odd. Then $\omega(G)=\left[\left(q^{2}+1\right) / 2\right]$.

Proof By Theorem 2.6, we have $\left[\left(q^{2}+1\right) / 2\right]=\omega(X) \leq \omega(G)$. Let $\Omega$ be a set of elements of $G$ which defines a clique in $\Gamma(G)$. It is sufficient to show that $|\Omega| \leq$ $\left[\left(q^{2}+1\right) / 2\right]$.

When $q$ is even $\operatorname{SL}(2, q) \cong \operatorname{PSL}(2, q)$ and $\operatorname{GL}(2, q) \cong \operatorname{SL}(2, q) \times C_{q-1}$ (so $\operatorname{PGL}(2, q) \cong \operatorname{SL}(2, q))$. Hence, in this case, it is sufficient to assume that $G=$ $\operatorname{PSL}(2, q)$.

The intersection of $\Omega$ with any member of $\Sigma$ is either empty or has size 1 . The elements $g$ of $G$ fall into three categories. The element $g$ is irreducible and so it is in a Singer cycle. In this case $g$ is in exactly one member of $\Sigma$. Let $\alpha$ denote the number of irreducible elements in $\Omega$. Then $\alpha \leq t=q(q-1) / 2$. Secondly, the element $g$ does not lie in a Singer cycle and exactly one member of $\Sigma$ contains $g$. (In this case, $g$ is contained in $P_{q+1}$ (when $q$ is even) or $g$ is contained in the maximal subgroup $M$ of $G$ with (P) $\operatorname{SL}(2, q)<M$ and $[G: M]=2$ (when $q$ is odd).) Let the number of such elements $g$ in $\Omega$ be $\beta$. Clearly, $\beta \leq 1$. Thirdly, the element $g$ is contained in at least two members of $\Sigma$. The number of such elements in $\Omega$ is at most $[((q(q+1) / 2)+\epsilon-\alpha-\beta) / 2]$ where $\epsilon=0$ when $q$ is even and $\epsilon=1$ when $q$ is odd. Hence we get

$$
\begin{aligned}
|\Omega| & \leq \alpha+\beta+\left[\frac{(q(q+1) / 2)+\epsilon-\alpha-\beta}{2}\right]=\left[\frac{(q(q+1) / 2)+\epsilon+\alpha+\beta}{2}\right] \\
& \leq\left[\frac{q^{2}+1}{2}\right] .
\end{aligned}
$$

\section{Suzuki groups}

In this section let $G$ be the Suzuki group $\operatorname{Suz}(q) \leq \operatorname{GL}(4, q)$ where $q=2^{2 m+1}$ for some positive integer $m$ at least 2 . We already know the covering number $\sigma(G)$ of $G$.

Theorem 3.1 (Lucido, [18]) Let $G=\operatorname{Suz}(q)$. Then $\sigma(G)=q^{2}\left(q^{2}+1\right) / 2$.

The purpose of this section is to show

Theorem 3.2 Let $G=\operatorname{Suz}(q)$. Then $\omega(G)=q^{4} / 2$ and $\chi(G)=\left(q^{2}\left(q^{2}+1\right) / 2\right)-1$.

Proof The order of $G$ is $q^{2}(q-1)\left(q^{2}+1\right)$. The integer $q^{2}+1$ can be factorized as $(q-r+1)(q+r+1)$ where $r=2^{m+1}$. If $U$ is the subgroup of the lower unitriangular matrices of $G$, then $U$ is a Sylow 2-subgroup of exponent 4 and of order $q^{2}$. Let $H$ be the subgroup of the diagonal matrices of $G$. Then $H$ is isomorphic to the multiplicative group of the field, and therefore has order $q-1$; it is a $\pi(q-1)$-Hall subgroup of $G$ and it normalizes $U$. For $i=1,2$, let $T_{i}$ be a (cyclic) maximal torus of order $q+(-1)^{i} r+1$. Let $\varphi$ be the set of all conjugates of the subgroups $U, H, T_{1}$, and $T_{2}$. Then, by Theorem 3.10, Chapter XI of [13], we see that $\varphi$ is a partition of $G$, 
that is, every non-identity element of $G$ is contained in exactly one member of $\varphi$. By [24], the only maximal subgroup of $G$ containing $T_{i}$ is $N_{i}=N_{G}\left(T_{i}\right)=T_{i}\left\langle t_{i}\right\rangle$, with $t_{i}$ an element of order 4 and $\left|N_{i}: T_{i}\right|=4$, for $i=1,2$. The 2-elements of $G$ are contained in the union of the conjugates of $N_{1}$. By [24], there are two kinds of maximal subgroups containing $H$ : some conjugates of the Borel subgroup $B$ of $G$ defined by $N_{G}(U)=U H$, and some conjugates of the subgroup $N_{G}(H)=H\langle t\rangle$ where $t \notin U$ is an involution. Since $B$ is a Frobenius group, there are $q^{2}$ conjugates of $H$ in $B$, and since $\left|N_{G}(H)\right|=2|H|$, there are $q^{2}\left(q^{2}+1\right) / 2$ conjugates of $H$ in $G$. We now want to examine the intersection of the Borel subgroup $B$ with its conjugates. To do this we need two facts from [13]. (i) $B \cap B^{t}=H$; (ii) any element $g$ of $G \backslash B$ can be written uniquely in the form $g=b t u$ with $b \in B$ and $u \in U$. Therefore, if $g \in G \backslash B$, we have $B \cap B^{g}=H^{u}$, where $u$ is the unique element of $U$ such that $g=b t u$. It follows that if $B^{g_{1}} \neq B^{g_{2}}$, then $B \cap B^{g_{1}} \neq B \cap B^{g_{2}}$. Therefore there are $q^{2}+\left(q^{2}-1\right)$ conjugates of $H$ in $B \cup B^{g}$ provided that $g \in G \backslash B$.

There are $q^{2}\left(q^{2}-1\right) / 2$ conjugates of $T_{1}$ and $T_{2}$ in $G$. Pick a generator from each of these subgroups. Let these be $s_{1}, \ldots, s_{q^{2}\left(q^{2}-1\right) / 2}$. There are $q^{2}+1$ Borel subgroups in $G$. Let these be $B_{1}, \ldots, B_{q^{2}+1}$. For each pair of indices $i$ and $j$ with $1 \leq i<j \leq q^{2}+1$, let $k_{i, j}$ be a generator of $B_{i} \cap B_{j}$. Let $\widetilde{X}$ be the induced subgraph of $\Gamma(G)$ spanned by the set consisting of all the $s_{i}$ 's and all the $k_{i, j}$ 's. Notice that the graph $\widetilde{X}$ is isomorphic to the graph $X$ defined before the statement of Theorem 2.6 with the only modification that we replace the even prime power $q$ (at least 4) by $q^{2}$. Hence the first two paragraphs of the proof of Theorem 2.6 can be used to show that $\omega(\tilde{X})=q^{4} / 2$ and $\chi(\tilde{X})=\left(q^{2}\left(q^{2}+1\right) / 2\right)-1$.

Let $\Sigma$ be the set of all conjugates of all normalizers of $T_{1}$ and $T_{2}$ together with all the $B_{i}$ 's except $B_{q^{2}+1}$. The set $\Sigma$ is a covering for $G$. Put $\widetilde{\Sigma}=\left(\Sigma \backslash\left\{B_{q^{2}-1}, B_{q^{2}}\right\}\right) \cup$ $\{B\}$ where $B$ is the set of all powers of the elements $k_{q^{2}-1, q^{2}}, k_{q^{2}, q^{2}+1}, k_{q^{2}-1, q^{2}+1}$. Clearly, $|\widetilde{\Sigma}|=\left(q^{2}\left(q^{2}+1\right) / 2\right)-1$, every element of $G$ is contained in some member of $\widetilde{\Sigma}$, and every member of $\widetilde{\Sigma}$ induces an empty subgraph in $\Gamma(G)$. This proves $\chi(G) \geq\left(q^{2}\left(q^{2}+1\right) / 2\right)-1$. The other inequality follows from $\chi(\tilde{X}) \leq \chi(G)$.

In order to complete the proof of the theorem we only need to show that $\omega(G) \leq$ $q^{4} / 2$. Let $\Omega$ be a subset of $G$ that defines a clique in $\Gamma(G)$. The intersection of $\Omega$ with any member of $\Sigma$ is either empty or has size 1 . The elements $g$ of $G$ fall into three categories. The element $g$ may lie in a unique conjugate of $T_{1}$ or $T_{2}$. In this case $g$ is in exactly one member of $\Sigma$. Let $\alpha$ denote the number of such elements in $\Omega$. Secondly, the element $g$ does not lie in any conjugate of $T_{1}$ or $T_{2}$ and exactly one member of $\Sigma$ contains $g$. (In this case $g$ is contained in $B_{q+1}$.) Let the number of such elements $g$ in $\Omega$ be $\beta$. Clearly, $\beta \leq 1$. Thirdly, the element $g$ is contained in at least two members of $\Sigma$. The end of the proof of Theorem 2.7 may now be applied to reach the desired conclusion.

\section{Ree groups}

Apart from an explicit finite list of exceptions, we found that whenever $G$ is any of the groups $\operatorname{GL}(2, q), \operatorname{SL}(2, q), \operatorname{PGL}(2, q), \operatorname{PSL}(2, q), \operatorname{Suz}(q)$, then $1-1 / m(G) \leq$ 
$\omega(G) / \sigma(G)$ where $m(G)$ denotes the minimal index of a proper subgroup in $G$. In this section we prove a similar result for Ree groups.

Let $G=\operatorname{Ree}(q)$ be the Ree group where $q=3^{2 m+1}$ for some positive integer $m$ at least 2 .

Theorem 4.1 Let $G=\operatorname{Ree}(q)$. Then we have $1-4 /\left(q^{3}+1\right)<\omega(G) / \sigma(G)$.

Proof The size of $G$ is $q^{3}(q-1)\left(q^{3}+1\right)$, and we have $(q-r+1)(q+r+1)=$ $q^{2}-q+1$ where $r=3^{m+1}$. The maximal subgroups of $G$ are given on Page 61 of [14]. In [19] it is shown that the union of all conjugates of the (maximal) parabolic subgroups $\left[q^{3}\right]: Z_{q-1}$, all conjugates of $2 \times \operatorname{PSL}(2, q)$, all conjugates of $Z_{q+r+1}: Z_{6}$, and all conjugates of $Z_{q-r+1}: Z_{6}$ is $G$. (For more details on these maximal subgroups see [14].) This gives an upper bound of

$$
A(q):=\frac{q^{3}\left(q^{3}+1\right)(q-1)}{6(q+r+1)}+\frac{q^{3}\left(q^{3}+1\right)(q-1)}{6(q-r+1)}+\left(q^{3}+1\right)+\frac{q^{2}\left(q^{3}+1\right)}{(q+1)}
$$

for $\sigma(G)$. Now pick an element of order $q+r+1$ from each conjugate of the maximal subgroup $Z_{q+r+1}: Z_{6}$, an element of order $q-r+1$ from each conjugate of the maximal subgroup $Z_{q-r+1}: Z_{6}$, and an element of order $(q+1) / 2$ from each conjugate of $2 \times \operatorname{PSL}(2, q)$. Let the set consisting of these elements be $\Omega$. This defines a clique in $\Gamma(G)$ by the list of maximal subgroups of $G$ found in [14], and from the proof of Lemma 2.2 of [19]. The size of $\Omega$ is

$$
B(q):=\frac{q^{3}\left(q^{3}+1\right)(q-1)}{6(q+r+1)}+\frac{q^{3}\left(q^{3}+1\right)(q-1)}{6(q-r+1)}+\frac{q^{2}\left(q^{3}+1\right)}{(q+1)} .
$$

Careful calculation gives

$$
1-\frac{4}{q^{3}+1}<\frac{A(q)}{B(q)} \leq \frac{\omega(G)}{\sigma(G)}
$$

Note that $q^{3}+1$ is the minimal index of a proper subgroup in $G$.

\section{Linear groups of any dimension}

Throughout this section for any positive integer $n$ at least 2 and any prime power $q$ let $G$ be any of the groups $\operatorname{GL}(n, q), \operatorname{SL}(n, q), \operatorname{PGL}(n, q), \operatorname{PSL}(n, q)$.

By [3], exact formulas are known for $\omega(G)$ and $\sigma(G)$ provided that $n \geq 12$. In general (for any $n$ ) we only have estimates.

Theorem 5.1 (Corollary 6.1 of [3]) When $G=(\mathrm{P}) \operatorname{SL}(n, q)$ suppose that $n \geq 2$ and $(n, q) \neq(2,5),(2,7),(2,9),(3,4)$. Then

$|\mathrm{GL}(n, q)| /\left(\sum\left|\mathrm{GL}\left(n / a, q^{a}\right) \cdot a\right|\right) \leq \mu(G) \leq \sigma(G) \leq \frac{|\mathrm{GL}(n, q)|}{\left|\mathrm{GL}\left(n / b, q^{b}\right) \cdot b\right|}+\sum_{\substack{k=1 \\ b \nmid k}}^{[n / 2]}\left[\begin{array}{l}n \\ k\end{array}\right]_{q}$, 
where the first sum is over all prime divisors $a$ of $n$ and $b$ is the smallest prime divisor of $n$.

The observation of this section is the following.

Theorem 5.2 Let $G$ be any of the groups $(\mathrm{P}) \operatorname{SL}(n, q)$. Then, apart from finitely many groups $G$, we have $1-1 / m(G) \leq \omega(G) / \sigma(G)$ where $m(G)$ denotes the minimal index of a proper subgroup in $G$.

Proof By the section on linear groups of dimension 2, it is sufficient to assume that $n \geq 3$. Tedious calculations using the bounds in Theorem 5.1 give $1-(q-1) /\left(q^{n}-\right.$ $1) \leq \omega(G) / \sigma(G)$ provided that $n \geq 3$ and $(n, q) \neq(3,2),(3,3),(3,4)$.

This completes the proof of Theorem 1.1.

\section{Linear groups of large dimensions}

In this section let $G$ be any of the groups $\operatorname{GL}(n, q), \operatorname{SL}(n, q), \operatorname{PGL}(n, q), \operatorname{PSL}(n, q)$. Suppose also that $n \geq 12$.

We aim to determine $\chi(G)$. Clearly, $\omega(G) \leq \chi(G) \leq \sigma(G)$. By [3], we have formulas for $\omega(G)$ and $\sigma(G)$ which are close to each other.

By Theorem 1.2 of [3], we may assume that $n \equiv 2(\bmod 4), q$ odd and $G=$ (P) GL $(n, q)$, or $n \equiv 2(\bmod 4)$ and $q$ even. (For otherwise $\omega(G)=\sigma(G)$ and so $\omega(G)=\chi(G)=\sigma(G)$.) Also, since $\chi(G) \leq \chi(G / Z(G)), \omega(G)=\omega(G / Z(G))$ and $\sigma(G)=\sigma(G / Z(G))$, it is sufficient to assume that $G=\operatorname{GL}(n, q)$ or $G=\operatorname{SL}(n, q)$.

Let $V$ be an $n$-dimensional vector space over the field of $q$ elements on which $G$ acts naturally. Put $t=|\operatorname{GL}(n, q)| /\left|\operatorname{GL}\left(n / 2, q^{2}\right) .2\right|$. Let $U_{1}, \ldots, U_{\ell}$ be a list of all subspaces of $V$ of odd dimensions less than $n / 2$ and let $W_{1}, \ldots, W_{\ell}$ be a list of all subspaces of $V$ of odd dimensions greater than $n / 2$ listed in an order such that $V=U_{i} \oplus W_{i}$ for all $i$ with $1 \leq i \leq \ell$. (Such an ordering of subspaces is always possible for example by the second paragraph of Section 3 of [3].) Let $V_{1}, \ldots, V_{r}$ be a list of all $n / 2$-dimensional subspaces of $V$. The letter $g$ with some subscript(s) will always denote an element of $G$. For a positive integer $i$ the element $g_{i}$ will denote an irreducible element in $G$. For subspaces $U_{i}, W_{i}$ the element $g_{U_{i}, W_{i}}$ will denote an element of $G$ that leaves exactly two proper, non-trivial subspaces of $V$ invariant, namely $U_{i}$ and $W_{i}$. When $q$ is odd, then let $g_{V_{i}}$ denote an element of $G$ leaving exactly one proper, non-trivial subspace of $V$ invariant, namely $V_{i}$. Finally, for complementary subspaces $V_{i}$ and $V_{j}$ an element of $G$ leaving exactly two proper, non-trivial subspaces of $V$ invariant, namely $V_{i}$ and $V_{j}$, shall be denoted by $g_{\left\{V_{i}, V_{j}\right\}}$.

Recall that we are assuming that $n \geq 12$. By [3], there exists a subset

$$
\Sigma=\left\{g_{i}\right\}_{i=1}^{t} \cup\left\{g_{U_{i}, W_{i}}\right\}_{i=1}^{\ell} \cup\left\{g_{V_{i}}\right\}_{i=1}^{r} \cup\left\{g_{\left\{V_{i}, V_{j}\right.}\right\}_{V=V_{i} \oplus V_{j}}
$$

so that distinct elements $x, y \in \Sigma$ generate $G$ if and only if they do not leave the same proper, non-trivial subspace of $V$ invariant unless $x=g_{V_{i}}$ and $y=g_{V_{j}}$ for some $i$ and 
$j$ when $\langle x, y\rangle$ is a subgroup of a group of index 2 in $G$ (this occurs only when $q$ is odd).

Now let $\Sigma$ denote also the induced subgraph of $\Gamma(G)$ spanned by the set $\Sigma$. Let $\chi(\Sigma)$ denote the chromatic number of the graph $\Sigma$. Clearly, $\chi(\Sigma) \leq \chi(G) \leq \sigma(G)$.

Put $\Sigma_{1}=\left\{g_{i}\right\}_{i=1}^{t} \cup\left\{g_{U_{i}, W_{i}}\right\}_{i=1}^{\ell}$ and $\Sigma_{2}=\left\{g_{V_{i}}\right\}_{i=1}^{r} \cup\left\{g_{\left\{V_{i}, V_{j}\right\}}\right\}_{V=V_{i} \oplus V_{j}}$. Similarly, let $\Sigma_{1}$ and $\Sigma_{2}$ denote the induced subgraphs of $\Sigma$ spanned by the sets $\Sigma_{1}$ and $\Sigma_{2}$ respectively. Then $\Sigma_{1}$ is a complete subgraph and every vertex on $\Sigma_{1}$ is connected to every vertex in $\Sigma_{2}$. Hence $\chi(\Sigma)=\left|\Sigma_{1}\right|+\chi\left(\Sigma_{2}\right)$. By this observation and by Theorem 1.2 of [3] the following question may be of interest.

Question 6.1 Is it true that $\chi\left(\Sigma_{2}\right)=\left(q^{n / 2} /\left(q^{n / 2}+1\right)\right)\left[\begin{array}{c}n \\ n / 2\end{array}\right]_{q}+\epsilon$ where $\epsilon$ is 0 if $q$ is even and 1 if $q$ is odd?

An affirmative answer would imply $\chi(\Sigma)=\chi(G)=\sigma(G)$.

Let $\Sigma_{2}^{\prime}$ be the set $\left\{g_{\left\{V_{i}, V_{j}\right\}}\right\}_{V=V_{i} \oplus V_{j}}$. Let $\Sigma_{2}^{\prime}$ also denote the associated induced subgraph in $\Gamma(G)$. We aim to give a lower bound for $\chi\left(\Sigma_{2}^{\prime}\right)$ for any $q$ (either even or odd).

Let $s$ be $\left(1 /\left(q^{n / 2}+1\right)\right)\left[\begin{array}{c}n \\ n / 2\end{array}\right]_{q}$ and let $m$ be a positive integer with $s<m \leq r$ where $r=\left[\begin{array}{c}n \\ n / 2\end{array}\right]_{q}$.

Lemma 6.1 Let $\Delta$ be a graph on $m$ vertices so that every subset of $s+1$ vertices spans a non-empty induced subgraph in $\Delta$. Then the number of edges in $\Delta$ is at least $(m / 2)((m / s)-1)$.

Proof Let $\bar{\Delta}$ be the complementary graph of $\Delta$. (There is an edge in $\bar{\Delta}$ between two given distinct vertices iff there is no edge between those vertices in $\Delta$.) By our hypothesis, $\bar{\Delta}$ does not contain a complete subgraph on $s+1$ vertices, and hence, by Turán's theorem [26], contains at most $(1-(1 / s))\left(m^{2} / 2\right)$ edges.

The graph $\Sigma_{2}^{\prime}$ is the union of $\chi\left(\Sigma_{2}^{\prime}\right)$ color classes, that is, empty subgraphs. What are the maximal empty induced subgraphs of $\Sigma_{2}^{\prime}$ ? They are of two kinds: (1) for every $n / 2$-dimensional vector space $V_{i}$ the set $A_{i}=\left\{g_{\left\{V_{i}, V_{j}\right\}}\right\}_{j}$ where $j$ runs through the integers for which $V_{i} \cap V_{j}=\{0\}$; (2) for every $n / 2$-dimensional vector spaces $V_{i}, V_{j}, V_{k}$ with $V_{i} \cap V_{j}=V_{j} \cap V_{k}=V_{k} \cap V_{i}=\{0\}$ the set $A_{i, j, k}=$ $\left\{g_{\left\{V_{i}, V_{j}\right\}}, g_{\left\{V_{j}, V_{k}\right\}}, g_{\left\{V_{k}, V_{i}\right\}}\right\}$.

Lemma 6.2 We have $\chi\left(\Sigma_{2}^{\prime}\right) \leq r-s$.

Proof Let $v$ be a fixed non-zero vector in $V$. Let $\mathcal{C}_{v}$ be the set of those $A_{i}$ 's for which $v \notin V_{i}$. Then $\mathcal{C}_{v}$ is a vertex-covering of the graph $\Sigma_{2}^{\prime}$ using $r-s$ maximal empty induced subgraphs.

Let $\mathcal{C}$ be a vertex covering of the graph $\Sigma_{2}^{\prime}$ using sets of type (1) and (2). Suppose that the number of sets of type (1) in $\mathcal{C}$ is $\alpha$ and the number of sets of type (2) in $\mathcal{C}$ is $\beta$. Suppose that $\alpha+\beta=\chi\left(\Sigma_{2}^{\prime}\right)$. 
Lemma 6.3 We have $r-6 s \leq \alpha \leq \chi\left(\Sigma_{2}^{\prime}\right)$.

Proof Let $\Delta$ be the set of $n / 2$-dimensional subspaces of $V$ with the property that $V_{i} \in$ $\Delta$ if and only if $A_{i} \notin \mathcal{C}$. Let $m$ be the size of $\Delta$. Clearly, $m=r-\alpha$. Let $\Delta$ denote also the induced subgraph of the $q$-Kneser graph $q K(n, n / 2)$ (defined in the Introduction) spanned by the set $\Delta$. By our construction, the edge set of the graph $\Delta$ is contained in the union of $\beta$ triangles. By a result of Frankl and Wilson [10], a 1-intersecting family of $n / 2$-dimensional subspaces of $V$ has size at most $s$. (A set $\mathcal{V}$ of $n / 2$-dimensional subspaces of $V$ is called a 1-intersecting family if for any distinct vector spaces $V_{i}$ and $V_{j}$ from $\mathcal{V}$ we have $V_{i} \cap V_{j} \neq\{0\}$.) This implies that $\Delta$ does not contain an empty induced subgraph on $s+1$ vertices. Hence, by Lemma 6.1, the number of edges in $\Delta$ is at least $(m / 2)((m / s)-1)$. Now $3(m-s)<(m / 2)((m / s)-1)$ provided that $m>6 s$. So if, for a contradiction, we had $\alpha<r-6 s$, then $m-s<\beta$ and so $r-m+m-s<\alpha+\beta=\chi\left(\Sigma_{2}^{\prime}\right)$ contradicting Lemma 6.2.

Now let $\Sigma^{\prime}$ be the set $\Sigma_{1} \cup \Sigma_{2}^{\prime}$. Let $\Sigma^{\prime}$ also denote the induced subgraph of $\Gamma(G)$ spanned by the set $\Sigma^{\prime}$. Clearly, $\chi\left(\Sigma^{\prime}\right)=\left|\Sigma_{1}\right|+\chi\left(\Sigma_{2}^{\prime}\right)=t+\ell+\chi\left(\Sigma_{2}^{\prime}\right)$ as $\Sigma_{1}$ is a complete subgraph and every vertex of $\Sigma_{1}$ is connected to every vertex of $\Sigma_{2}^{\prime}$. Finally, by Lemma 6.3, we have $t+\ell+r-6 s \leq \chi\left(\Sigma^{\prime}\right) \leq \chi(G)$.

Comparing this lower bound for $\chi(G)$ with the formulas of Theorem 1.1 and Theorem 1.2 of [3] we have

$$
\begin{aligned}
& \omega(G) \geq \frac{1}{2} \prod_{\substack{i=1 \\
2 \nmid i}}^{n-1}\left(q^{n}-q^{i}\right)+\sum_{\substack{k=1 \\
2 \nmid k}}^{(n / 2)-1}\left[\begin{array}{l}
n \\
k
\end{array}\right]_{q}+\frac{1}{2}\left[\begin{array}{c}
n \\
n / 2
\end{array}\right]_{q}-1 ; \\
& \sigma(G)=\frac{1}{2} \prod_{\substack{i=1 \\
2 \nmid i}}^{n-1}\left(q^{n}-q^{i}\right)+\sum_{\substack{k=1 \\
2 \nmid k}}^{(n / 2)-1}\left[\begin{array}{l}
n \\
k
\end{array}\right]_{q}+\frac{q^{n / 2}}{q^{n / 2}+1}\left[\begin{array}{c}
n \\
n / 2
\end{array}\right]_{q}+\epsilon ; \\
& \chi(G) \geq \frac{1}{2} \prod_{\substack{i=1 \\
2 \nmid i}}^{n-1}\left(q^{n}-q^{i}\right)+\sum_{\substack{k=1 \\
2 \nmid k}}^{(n / 2)-1}\left[\begin{array}{l}
n \\
k
\end{array}\right]_{q}+\frac{q^{n / 2}-5}{q^{n / 2}+1}\left[\begin{array}{c}
n \\
n / 2
\end{array}\right]_{q}
\end{aligned}
$$

where $\epsilon$ is 1 if $q$ is odd and is 0 if $q$ is even.

From the latter three formulas Theorem 1.2 follows.

\section{Kneser graphs as induced subgraphs}

In this section let $n$ and $r$ be positive integers with $r<n / 2$. Let $\Omega$ be a set of size $n$ and let $\Omega_{1}, \ldots, \Omega_{t}$ be the distinct $r$-subsets of $\Omega$ where $t=\left(\begin{array}{l}n \\ r\end{array}\right)$. For each $i$ with $1 \leq i \leq t$ let $V_{i}$ be the set of all permutations of $\operatorname{Sym}(\Omega)$ which fix every element of $\Omega_{i}$ and permute all the elements of $\Omega \backslash \Omega_{i}$ in an $(n-r)$-cycle. Let $\Gamma$ be the graph whose vertex-set is $V=V_{1} \cup \ldots \cup V_{t}$ and there is an edge between two vertices if and only if no point-stabilizer contains both elements and they do not generate a group 
containing $\operatorname{Alt}(\Omega)$. To prove Theorem 1.3 it is sufficient to find an independent set $\left\{v_{1}, \ldots, v_{t}\right\}$ in $V=V(\Gamma)$ so that $v_{i} \in V_{i}$ for each $i$ with $1 \leq i \leq t$.

By the following result (see also [12] and [22]), it is sufficient to show that 2 times the degree of any vertex in $\Gamma$ is at most $(n-r-1)$ !.

Theorem 7.1 (Haxell, [11]) Let $\Gamma$ be a (simple) graph so that every vertex of $\Gamma$ has degree at most d for some positive integer d. Let $V(\Gamma)=V_{1} \cup \ldots \cup V_{t}$ be a partition of the vertex set of $\Gamma$. Suppose that $2 d \leq\left|V_{i}\right|$ for each $i$. Then $\Gamma$ has an independent set $\left\{v_{1}, \ldots, v_{t}\right\}$ where $v_{i} \in V_{i}$ for each $i$.

Let $v$ be a fixed vertex in $V$ and let $d$ be its degree in $\Gamma$. It is sufficient to show that $2 d \leq\left|V_{1}\right|=(n-r-1)$ !.

If $\{v, w\}$ is an edge in $\Gamma$, then $v$ and $w$ generate a transitive subgroup of $\operatorname{Sym}(\Omega)$ not containing $\operatorname{Alt}(\Omega)$. (This is because we are assuming that $r<n / 2$.) Hence there are two possibilities: $\langle v, w\rangle$ is contained in a maximal primitive group different from $\operatorname{Alt}(n)$, or $\langle v, w\rangle$ is contained in a maximal imprimitive subgroup of $\operatorname{Sym}(\Omega)$.

Let $H$ be a maximal subgroup in $\operatorname{Sym}(\Omega)$. Let $h$ be the number of conjugates of $H$ containing a fixed element of $V$. (Notice that $h$ is well-defined since $V$ is a conjugacy class of elements in $\operatorname{Sym}(n)$.) The number of ordered pairs $(u, K)$ such that $u \in V$, the subgroup $K$ is conjugate to $H$, and $u \in K$ is equal to $(h n !) /((n-r) r !)$ and less than $(n ! /|H|)|H|=n$ !. Hence $h<(n-r) r$ !.

Let $H$ be a maximal imprimitive subgroup of $\operatorname{Sym}(\Omega)$. Then it is easy to see that $|H \cap V|<2^{n / 2}\left(2([(n-r) / 2] !)^{2}\right) /(n-r)$. For a maximal primitive subgroup $H$ not containing $\operatorname{Alt}(\Omega)$ we have $|H \cap V|<|H| \leq 3^{n}$ by [20].

By [15], there are at most $o(1) n$ conjugacy classes of transitive maximal subgroups in $\operatorname{Sym}(\Omega)$.

Hence, for a fixed $r$, the number of neighbors of $v$ in $\Gamma$ is

$$
d<o(1) r ! 2^{(n / 2)+1} n([(n-r) / 2] !)^{2} .
$$

Since

$$
\frac{2^{n-r-1}}{n-r+1}<\frac{(n-r) !}{2([(n-r) / 2] !)^{2}}
$$

we have

$$
d<o(1) r ! 2^{-(n / 2)+r+1} n(n-r+1) !<\left(o(1) r^{r} n^{3} 2^{-(n / 2)+r+2}\right) \frac{\left|V_{1}\right|}{2},
$$

where the second inequality followed from $r ! \leq r^{r}$ and

$$
(n-r+1) !<n^{2}(n-r-1) !=n^{2}\left|V_{1}\right| .
$$

There exists a universal constant $c$ so that whenever $2 r \log r+2 r+c<n$, then $o(1) r^{r} n^{3} 2^{-(n / 2)+r+2}<1$. Hence $d<\left|V_{1}\right| / 2$ provided that $2 r \log r+2 r+c<n$. This completes the proof of Theorem 1.3. 


\section{Two consequences of a theorem of Liebeck and Shalev}

For a finite group $G$ let $P(G)$ denote the probability that two randomly chosen elements from $G$ generate $G$. Let $m(G)$ be the minimal index of a proper subgroup in $G$. We begin with

Theorem 8.1 (Liebeck, Shalev, [16]) There exist constants $c_{1}, c_{2}>0$ such that

$$
1-\frac{c_{1}}{m(G)} \leq P(G) \leq 1-\frac{c_{2}}{m(G)}
$$

for all non-abelian finite simple groups $G$. Moreover, we have $\liminf m(G)(1-$ $P(G))=1$ and $\lim \sup m(G)(1-P(G))=3$ where the limits are taken as $G$ ranges over all non-abelian finite simple groups.

We will present an application (Theorem 1.4) of the upper bound of Theorem 8.1 and an application (Theorem 1.5) of the lower bound of Theorem 8.1.

We continue with the following observation.

Lemma 8.1 Let $G$ be a finite group. For a proper subgroup $H$ of index $m$ in $G$ we have $P(G) \leq 1-\left(1 / \mathrm{m}^{2}\right)$ and for a normal subgroup $N$ of $G$ we have $P(G) \leq$ $P(G / N)$.

Proof The first claim is clear since $P(G) \leq 1-\left(|H|^{2} /|G|^{2}\right)=1-\left(1 / m^{2}\right)$. The second claim follows from

$$
\begin{aligned}
|G|^{2} P(G) & =\left|\left\{(g, h) \in G^{2}:\langle g, h\rangle=G\right\}\right| \leq\left|\left\{(g, h) \in G^{2}:\langle g N, h N\rangle=G / N\right\}\right| \\
& =|N|^{2}\left|\left\{(g N, h N) \in(G / N)^{2}:\langle g N, h N\rangle=G / N\right\}\right|=|G|^{2} P(G / N) .
\end{aligned}
$$

Note that if $G$ is not solvable then it admits a monolithic group with non-abelian socle as an epimorphic image. By Lemma 8.1, to show Theorem 1.4, it is sufficient to assume that $G$ is a monolithic primitive group with $\operatorname{Soc}(G) \cong S^{t}$ for some nonabelian finite simple group $S$ and positive integer $t$. Clearly $G$ has a subgroup of index $t$ and $60^{t} \leq|S|^{t} \leq|G|$. We may assume that $t=1$. For if $t>1$, then, by Lemma 8.1, we have

$$
P(G) \leq 1-\left(1 / t^{2}\right) \leq 1-\left(1 / 60^{t / 3}\right) \leq 1-\left(1 /|G|^{1 / 3}\right)
$$

Now $G / S$ is isomorphic to a subgroup of $\operatorname{Out}(S)$ and so there exists a positive constant $d_{1}$ with $|G / S|^{2} \leq\left(1 / d_{1}\right)|G|^{1 / 3}$. By Lemma 8.1, $P(G) \leq 1-\left(d_{1} /|G|^{1 / 3}\right)$ provided that $G \neq S$. Hence we may assume that $G=S$. Then there exists a positive constant $d_{2}$ so that $P(G) \leq 1-\left(c_{2} / m(G)\right) \leq 1-\left(d_{2} /|G|^{1 / 3}\right)$ where $c_{2}$ is as in Theorem 8.1. Finally, to establish Theorem 1.4, take $d$ to be the minimum of $d_{1}$ and $d_{2}$.

We now turn to the proof of Theorem 1.5. 
Let $K_{r}(t)$ be the complete $r$-partite graph with $t$ vertices in each class, or equivalently the Turán graph $T(r t, r)$. Define $s_{r, \epsilon}(n)$ (for $0<\epsilon<1 /(2(r-1))$ ) to be the greatest $t$ such that every graph of order $n$ and the integer part of

$$
\left(\frac{r-2}{2(r-1)}+\epsilon\right) n^{2}
$$

edges contains a $K_{r}(t)$. The graph $K_{r}(t)$ contains every $r$-colorable graph on $r t$ vertices. Erdős and Stone [9] found a weak lower bound for $s_{r, \epsilon}(n)$ for $n$ sufficiently large. The correct order of $s_{r, \epsilon}(n)$ in terms of $n$ was found by Bollobás and Erdős [2]: for any given $r$ and $\epsilon$ there are constants $k_{1}(r, \epsilon)$ and $k_{2}(r, \epsilon)$ such that $k_{1}(r, \epsilon) \log n<s_{r, \epsilon}(n)<k_{2}(r, \epsilon) \log n$. Chvátal and Szemerédi [6] then determined the nature of the dependence on $r$ and $\epsilon$, up to a constant:

$$
\frac{\log n}{500 \log (1 / \epsilon)}<s_{r, \epsilon}(n)<\frac{5 \log n}{\log (1 / \epsilon)}
$$

for sufficiently large $n$.

Let $c_{1}$ be as in Theorem 8.1. Let $d_{1,1}$ and $d_{2,1}$ be positive constants so that whenever $G$ is a non-abelian finite simple group with $m(G)<4 c_{1}$ then every $r$-colorable graph on at most $d_{2,1} m(G)(\log |G| / \log m(G))$ vertices is a subgraph of $\Gamma(G)$ for any $r \leq d_{1,1} m(G)$. (Note that this is possible since there are at most finitely many nonabelian finite simple groups $G$ with $m(G)<4 c_{1}$.) Let $d_{2,2}$ be a positive constant so that $d_{2,2} m(G)(\log |G| / \log m(G))<|G|$ for any non-abelian finite simple group $G$.

Let $G$ be a non-abelian finite simple group with $4 c_{1} \leq m(G)$. Let $n=|G|$. By Theorem 8.1, the number of edges in $\Gamma(G)$ is at least $\left(1-\left(c_{1} / m(G)\right)\right)\left(n^{2} / 2\right)$. Let $r$ be the integer part of $m(G) /\left(2 c_{1}\right)$ and let $\epsilon$ be $1 /(4(r-1))$. Then

$$
\left(\frac{r-2}{2(r-1)}+\epsilon\right) n^{2}<\left(1-\left(c_{1} / m(G)\right)\right) \frac{n^{2}}{2},
$$

and so $\Gamma(G)$ contains a $K_{r}(t)$ for $t \leq s_{r, \epsilon}(n)$. Hence $\Gamma(G)$ contains (as subgraphs) every $r$-colorable graph on at most

$$
\frac{r \log n}{500 \log (4(r-1))}
$$

vertices. Finally, there exist universal positive constants $d_{1,2}$ and $d_{2,3}$ (independent of $G)$ so that $d_{1,2} m(G) \leq r$ and

$$
d_{2,3} m(G) \frac{\log n}{\log m(G)} \leq \frac{r \log n}{500 \log (4(r-1))} .
$$

Now let $d_{1}$ be the minimum of $d_{1,1}$, and $d_{1,2}$ and let $d_{2}$ be the minimum of $d_{2,1}, d_{2,2}$, and $d_{2,3}$. One can see that $d_{1}$ and $d_{2}$ are suitable constants satisfying the statement of Theorem 1.5. 


\section{Finite simple groups}

In this section $G$ denotes a non-abelian finite simple group. Let $\alpha$ denote $\omega, \chi$, or $\sigma$. For a positive number $x$ define $\alpha(x)$ to be the number of positive integers $n$ at most $x$ with the property that there exists a non-abelian finite simple group $G$ so that $\alpha(G)=n$. In this section we prove

Theorem 9.1 $\alpha(x)=(2 \sqrt{2}+o(1))(\sqrt{x}) /(\ln x)$.

Let the minimal index of a proper subgroup in $G$ be denoted by $m(G)$. The proof of Theorem 9.1 depends on Theorem 1.5 and on the following corollary of results of Stringer.

Theorem 9.2 (Stringer, [23]) Apart from at most finitely many positive integers $n$ we have $n^{3}<\omega(\operatorname{Alt}(n))$.

For $i=0,1$, let $\alpha_{i}(x)$ be the number of integers $\alpha\left(G_{i}\right)$ so that $\alpha\left(G_{i}\right) \leq x$ where $G_{0}=\operatorname{PSL}(2, q)$ for some odd prime power $q$ and $G_{1}=\operatorname{PSL}(2, q), \operatorname{Suz}(q)$ for some even prime power $q, \operatorname{PSL}(3, q)$, or $\operatorname{Alt}(n)$. Let $\alpha_{2}(x)$ be the number of pairs $\left(d_{1}\right.$. $m(G), G)$ so that $d_{1} \cdot m(G) \leq x$ and $G \neq \operatorname{PSL}(2, q), \operatorname{PSL}(3, q), \operatorname{Alt}(n), \operatorname{Suz}(q)$ where $d_{1}$ is a constant from Theorem 1.5.

By the Prime Number Theorem and by Theorems 2.5, 2.7, 5.1, 3.2, 9.2, it follows that $\alpha_{0}(x)=(2 \sqrt{2}+o(1))(\sqrt{x}) /(\ln x)$ and that $\alpha_{1}(x)=(o(1) \sqrt{x}) /(\ln x)$. These observations together with Theorem 1.5 and $\alpha_{0}(x) \leq \alpha(x) \leq \sum_{i=0}^{2} \alpha_{i}(x)$ imply that to prove Theorem 9.1 it is sufficient to establish

Lemma 9.1 $\alpha_{2}(x)=(o(1) \sqrt{x}) /(\ln x)$.

Proof In counting all pairs $\left(d_{1} \cdot m(G), G\right)$ with $d_{1} \cdot m(G) \leq x$ it is sufficient to assume that $G$ is a Lie group different from $\operatorname{PSL}(2, q), \operatorname{PSL}(3, q), \operatorname{Suz}(q)$. (We may exclude the sporadic simple groups since there are only finitely many of them.)

The precise values of the minimal indices $m(G)$ of proper subgroups in the Lie groups $G$ can be found (for example) in Table 1 on page 60 of [7]. Every numerical entry of this table is either a positive integer, an infinite sequence depending on one variable, or a doubly infinite sequence depending on two variables. We may ignore all positive integer entries of Table 1 . Notice that all other numerical entries of Table 1 give rise to simply or to doubly infinite series of strictly increasing positive integers. Since there are only finitely many entries in the table, it is sufficient to verify that every numerical entry (or sequence) has $(o(1) \sqrt{x}) /(\ln x)$ members that are at most $x / d_{1}$.

Consider the numerical entry $m\left(\mathrm{G}_{n}(q)\right)$ associated to the Lie group $G=\mathrm{G}_{n}(q)$ where $n$ denotes the Lie rank (or twisted Lie rank) of $G$, the prime power $q$ is the size of the field over which $G$ is defined, and where $\mathrm{G}_{n}(q)$ stands for a single entry in Table 1 of [7]. It is easy to see by inspection that if $x$ is large enough and $m\left(\mathrm{G}_{n}(q)\right) \leq$ $x / c$, then $q \leq\left(x / d_{1}\right)^{1 / 3}$ and $n \leq \ln \left(x / d_{1}\right)$. Hence there are indeed $(o(1) \sqrt{x}) /(\ln x)$ pairs $\left(m\left(\mathrm{G}_{n}(q)\right), \mathrm{G}_{n}(q)\right)$ with $m\left(\mathrm{G}_{n}(q)\right) \leq n / d_{1}$. 


\section{References}

1. Blackburn, S.: Sets of permutations that generate the symmetric group pairwise. J. Combin. Theory Ser. A 113(7), 1572-1581 (2006)

2. Bollobás, B.: Erdős, P. On the structure of edge graphs. Bull. London Math. Soc. 5, 317-321 (1973)

3. Britnell, J.R., Evseev, A., Guralnick, R.M., Holmes, P.E., Maróti, A.: Sets of elements that pairwise generate a linear group. J. Combin. Theory Ser. A 115(3), 442-465 (2008)

4. Bryce, R.A., Fedri, V., Serena, L.: Subgroup coverings of some linear groups. Bull. Austral Math. Soc. 60(2), 227-238 (1999)

5. Chowdhury, A., Godsil, C., Royle, G.: Colouring lines in projective spaces. J. Combin. Theory Ser. A 113(1), 39-52 (2006)

6. Chvátal, V., Szemerédi, E.: On the Erdős-Stone theorem. J. London Math. Soc. 23(2), 207-214 (1981)

7. Damian, E., Lucchini, A.: On the Dirichlet polynomial of finite groups of Lie type. Rend. Sem. Mat. Univ. Padova, vol. 115 (2006)

8. Dickson, L.E.: Linear Groups: With an Exposition of the Galois Field Theory. Dover Publication Inc., New York (1958)

9. Erdôs, P., Stone, A.: On the structure of linear graphs. Bull. American Math. Soc. 52, 1087-1091 (1946)

10. Frankl, P., Wilson, R.M.: The Erdős-Ko-Rado theorem for vector spaces. J. Comb. Theory, Ser. A 43(2), 228-236 (1986)

11. Haxell, P.E.: A condition for matchability in hypergraphs. Graphs and Combinatorics 11, 245-248 (1995)

12. Haxell, P.E.: A note on vertex list colouring. Combin. Probab. Comput. 10(4), 345-347 (2001)

13. Huppert, B., Blackburn, N.: Finite Groups III. Springer, Berlin (1982)

14. Kleidman, P.B.: The maximal subgroups of the Chevalley groups $G_{2}(q)$, with $q$ odd, the Ree groups $G_{2}^{2}\left(q^{2}\right)$ and their automorphism groups. J. Algebra 117, 30-71 (1988)

15. Liebeck, M.W., Shalev, A.: Maximal subgroups of symmetric groups. J. Combin. Theory Ser. A 75, 341-352 (1996)

16. Liebeck, M.W., Shalev, A.: Simple groups, probabilistic methods, and a conjecture of Kantor and Lubotzky. J. Algebra 184(1), 31-57 (1996)

17. Lovász, L.: Kneser's conjecture, chromatic number, and homotopy. J. Combin. Theory, Ser. A 25 , 319-324 (1978)

18. Lucido, M.S.: On the covers of finite groups. In: Groups St Andrews 2001 in Oxford, Vol. II. London Math. Soc. Lecture Note Ser., vol. 305, pp. 395-399. Cambridge Univ. Press, Cambridge (2003)

19. Lucido, M.S.: On the $n$-covers of exceptional groups of Lie type. In: Groups St Andrews 2005, Vol. II. London Math. Soc. Lecture Note Ser., vol. 340, pp. 621-623. Cambridge Univ. Press, Cambridge (2007)

20. Maróti, A.: On the orders of primitive groups. J. Algebra 258(2), 631-640 (2002)

21. Maróti, A.: Covering the symmetric groups with proper subgroups. J. Combin. Theory Ser. A 110(1), 97-111 (2005)

22. Meshulam, R.: The clique complex and hypergraph matching. Combinatorica 21(1), 89-94 (2001)

23. Stringer, L.: Pairwise generating sets for the symmetric and alternating groups. PhD thesis, Royal Holloway, University of London (2008)

24. Suzuki, M.: On a class of doubly transitive groups. Ann. Math. 75, 105-145 (1962)

25. Tomkinson, M.J.: Groups as the union of proper subgroups. Math. Scand. 81, 191-198 (1997)

26. Turán, P.: An extremal problem in graph theory. Mat. Fiz. Lapok 48, 436-452 (1941) 